\title{
Photo-electric conversion in dye-doped nanocrystalline titania films
}

Hiromasa Nishikiori,* Yohei Uesugi, Nobuaki Tanaka, Tsuneo Fujii

Department of Environmental Science and Technology, Graduate School of Science and

Technology, Shinshu University, Nagano 380-8553, Japan

Corresponding author: Hiromasa Nishikiori

Tel: +81-26-269-5536

Fax: +81-26-269-5550

E-mail: nishiki@shinshu-u.ac.jp

Department of Environmental Science and Technology, Graduate School of Science and

Technology, Shinshu University, Nagano 380-8553, Japan 


\section{Abstract}

Influences of the titania nanostructure and dye dispersion in a dye-doped titania electrode on its photoelectric conversion property were investigated by simple spectroscopic and electric measurements. The dye-doped nanocrystalline titania electrodes were prepared on the glass plates coated with ITO and normal crystalline titania films by the following two procedures: (1) the dye-doped titania gel films were prepared from a titanium alkoxide solution containing the dye and then steam-treated, and (2) the titanium alkoxide sol containing the dye was refluxed and then spread onto the plates. The photocurrent quantum efficiency remarkably increased by the steam treatment and the reflux compared to that of the untreated dye-doped electrode consisting of amorphous titania gel. The efficiency in the former was higher than that in the latter. The growth and crystallization of the titania particles and the decrease in the defect density by these treatments improved the electric conductivity. The steam treatment was the more prominent method because it enhanced the electric conductivity of the titania depending on its nanostructure and the dye-titania interaction depending on the dye dispersion. These factors appear to play important roles in transport in the electron through the electrode.

Keywords: nanocrystal, titania, fluorescein, photo-electric conversion, sol-gel method. 


\section{Introduction}

Effective use of solar energy is one of the most attractive solutions of the energy and environmental problems. Dye-sensitized solar cells have been studied in detail and developed as a photosynthesis-mimetic photo-electric conversion method for the last two decades [1-3]. Fundamental understandings of such heterogeneous systems are required in various research fields. It is important to investigate the influence of the titania nanostructure and the interaction between the dye molecules and titania on the photo-electric conversion property of the dye-sensitized solar cells. Many scientists have investigated the electron donor-acceptor interaction between the dye molecules and the titania matrix [4-13]. We have studied the dye-doped titania gel, which is different from the conventional dye-adsorbed titania [14-17]. The dye-doped amorphous gel films are prepared without heating from a titanium alkoxide sol containing the dye molecules. The dye molecules exist in the nanopores of the gel. We interpret that the gel consists of the amorphous, nanosized, and particle-like units having the semiconductor-like quasi-conduction band structure with low density state [14-16]. Our previous studies indicated that the steam treatment of the dye-doped amorphous titania film remarkably improved the photo-electric conversion efficiency due to its crystallization $[14,15]$. The steam treatment leads to the formation of 
anatase-type titania nanocrystals having the conduction band with higher density and increases in the surface quality of the nanoparticles, which improves the anchoring geometry of the dye on their surface.

The characteristics of this system are a high dispersion of the dye and a high contact area between the dye and titania. The investigation of the dye-titania interaction in this system will result in determining new information expected to be applicable to conventional solar systems from a different viewpoint.

The preparation of titanium dioxide at low temperature using a wet process, such as the sol-gel method, has also been widely investigated because of the advantages of uniform nanoparticle formation and energy savings [18-27]. Reflux is also an effective method to crystallize the sol-gel system of the titanium alkoxide at low temperature $[21,24]$. The dye-doped titania nanoparticles can be obtained by refluxing the sol containing the dye.

In the previous studies, the dye-doped titania gel film was prepared on the ITO plate coated with normal crystalline titania layer, which worked as an electron transport mediator and promoted the electron injection from the dye-doped titania gel to the ITO [14,15]. This layer improved not only the physical adhesion between these layers, but also their electric junction. 
In this study, the dye-doped titania films were prepared by the following procedures on glass plates coated with ITO and nanocrystalline titania in order to obtain differences in the titania crystallinity and dye dispersion: (1) the dye-doped titania gel films were prepared from a titanium alkoxide solution containing the dye and then steam-treated, and (2) the titanium alkoxide sol containing the dye was refluxed and then spread onto the plates. Furthermore, the latter was steam-treated in order to clarify the difference between the influence of the steam treatment and reflux on the crystallization of the titania gel. On the basis of a comparison between the spectroscopic and photoelectric properties for the two systems, we investigated the effect of the titania nanostructure and the dye dispersion on the photo-electric property of the dye-doped titania films.

\section{Experimental}

\subsection{Materials}

Titanium tetraisopropoxide, ethanol, fluorescein, hydrochloric acid, nitric acid, diethylene glycol, iodine, and lithium iodide (Wako Pure Chemicals, S or a reagent grade) were used without further purification. Water was ion-exchanged and distilled. Glass plates coated with the ITO transparent electrode (AGC Fabritech) were soaked in

hydrochloric acid $\left(1.0 \mathrm{~mol} \mathrm{dm}^{-3}\right)$ for 2 hours and then rinsed with water. The 
electrolyte for the electrical measurement consisted of an diethylene glycol solution of iodine $\left(5.0 \times 10^{-2} \mathrm{~mol} \mathrm{dm}^{-3}\right)$ and lithium iodide $\left(0.50 \mathrm{~mol} \mathrm{dm}^{-3}\right)$.

\subsection{Preparation of electrodes}

The sol-gel reaction system was prepared by mixing $5.0 \mathrm{~cm}^{3}$ of titanium tetraisopropoxide, $25.0 \mathrm{~cm}^{3}$ of ethanol, $0.21 \mathrm{~cm}^{3}$ of water, and $0.21 \mathrm{~cm}^{3}$ of concentrated nitric acid as the catalyst of the sol-gel reaction and labeled SG-0. Fluorescein was dissolved into SG-0 in which the concentration of the fluorescein was $1.0 \times 10^{-2} \mathrm{~mol}$ $\mathrm{dm}^{-3}$ and this system labeled SG-f. The dip-coated thin films were made from the systems in which the sol-gel reaction proceeded for 1 day to prepare the electrodes.

In order to prepare the electrode samples coated with the crystalline titania, the glass plates with the ITO transparent electrode were dip-coated with the dye-free system (SG-0) and then heated at $500{ }^{\circ} \mathrm{C}$ for $30 \mathrm{~min}$. These electrodes were labeled E0. Furthermore, the working electrodes were prepared in the way in which the E0 was dip-coated with the sol-gel systems containing the fluorescein dye (SG-f).

The steam-treatment effect on the UV-visible absorption and photocurrent spectra of the electrode samples was investigated. Water was heated at $100{ }^{\circ} \mathrm{C}$ and the electrode samples were exposed to its steam for 2 hours. The pressure of the steam was about $100 \mathrm{kPa}$ [14-16]. The working electrode prepared from the SG-f and that 
steam-treated for $2 \mathrm{~h}$ were labeled WE and WE-s, respectively

The other sol-gel reaction system was prepared by mixing $30.0 \mathrm{~cm}^{3}$ of titanium tetraisopropoxide, $5.0 \mathrm{~cm}^{3}$ of ethanol, $180.0 \mathrm{~cm}^{3}$ of water, and $2.0 \mathrm{~cm}^{3}$ of concentrated nitric acid in order to examine the effect of refluxing the system on the spectroscopic and photoelectric properties of the electrode sample prepared from the system. Fluorescein was dissolved in this sol-gel system $\left(1.0 \times 10^{-2} \mathrm{~mol} \mathrm{dm}^{-3}\right)$ and refluxed at $80^{\circ} \mathrm{C}$ for $10 \mathrm{~h}$, which was labeled SG-fr [21,24]. The total volume of the SG-fr was much larger than that of SG-f because the larger reactor was used for reflux. The larger relative amount of water was required for complete hydrolysis of titanium tetraisopropoxide during reflux. The SG-fr was spread on the E0. The working electrode prepared from the SG-fr was also steam-treated under the above conditions in order to clarify the difference between the influence of the steam treatment and reflux on the crystallization of the titania gel and the spectroscopic and photoelectric properties of the electrode. The working electrode before and after the 2-h steam treatment were labeled WE-r and WE-rs, respectively.

\subsection{Measurements}

The layer thickness of the electrode samples was estimated from their cross section using a field emission scanning electron microscope (Hitachi S-4100). The 
UV-visible absorption spectra of the prepared electrode samples were observed using a spectrophotometer (Shimadzu UV-2500). The iodine-based electrolyte was allowed to soak into the space between the electrode sample and the counter Pt electrode. Monochromatic lights obtained from a fluorescence spectrophotometer (Shimazdu RF-5300) with a $150 \mathrm{~W}$ Xe short arc lamp (Ushio UXL-155) were irradiated on the electrodes for the spectroscopic measurements. Under light irradiation, the short circuit currents of the electrodes were measured by an electrometer (Keithley model 617). The $I-V$ curves of the electrodes were measured by a potentiostat (Hokuto Denko HSV-100) during irradiation by visible light with a wavelength longer than 400 nm emitted by the $150 \mathrm{~W}$ Xe short arc lamp with a sharp cut filter. The crystalline phase was determined using an X-ray diffractometer (Rigaku RINT-2200V). The intensity at each wavelength of the light source was obtained using a power meter (Molectron PM500A) in order to estimate the incident photon to current conversion efficiency (IPCE) and quantum efficiency for the photocurrent from the excited dye in the electrode samples. The light intensity was confirmed to correlate with the results of the potassium ferrioxalate actinometry. The absorbance of the present electrode samples was lower than 0.5 enough to measure the number of the adsorbed photon to calculate the quantum efficiency. 
3. Results and discussion

\subsection{Effects of steam treatment}

The absorption and IPCE spectra of the dye-doped titania gel electrode before (WE) and after the 2-h steam treatment (WE-s) are shown in Figure 1. The thickness of the dye-containing layer of WE and WE-s was ca. $350 \mathrm{~nm}$ and that slightly changed by the steam treatment. The WE exhibits a visible absorption spectrum that is located around $480 \mathrm{~nm}$, ranging over a wavelength longer than that observed in solvents. This result indicates that the main fluorescein species were the anion (at 450-480 $\mathrm{nm}$ ) and some fluorescein molecules existed as the dianion (at around $490 \mathrm{~nm}$ ) [28]. In addition, the longer wavelength band indicates that a small number of fluorescein molecules formed the dianion-like species resulting from the strong interaction and an ester-like or chelating linkage between the carboxyl group of the dye and the titanium species $[4,5,8-10,29,30]$. The carboxylate of fluorescein covalently bonding to the titania surface has been detected on by XPS analysis [29]. The absorbance decreased, the spectral peak of the film was red-shifted from around 480 to $485 \mathrm{~nm}$, and the absorption band appeared at $500-600 \mathrm{~nm}$ by the steam treatment $[14-16,30]$. These results indicate that the anion was preferentially desorbed from the inside of the titania 
gel film because the species was weakly trapped in the pores of the gel. The stream treatment probably increased the number of the fluorescein molecules interacting with the titanium species.

The change in the IPCE spectra of the fluorescein-doped titania-gel film by the steam treatment is also shown in Figure 1. Some of these values corresponded to the photocurrent data reported in a previous paper, even though the present values are grater than the previous ones due to a difference in the counter electrode $[14,15]$. In spite of the absorbance decrease, the photocurrent of the electrode increased with an increase in the treatment time while the peak at around $500 \mathrm{~nm}$ was only slightly changed. These results suggest that the increase in the IPCE values is due to improvement of the electric conductivity of the titania gel and the dianion-like species interacting with the titanium species (the around $500 \mathrm{~nm}$ species) plays an important role in generating the photocurrent in this system. The IPCE values were almost constant after a 3-h steam treatment $[14,15]$. The growth and crystallization of the particles and the decrease in the defect density by the steam treatment improved the electric conductivity.

\section{(Figure 1)}

\subsection{Effect of refluxing sol}

Figure 2 shows the visible absorption and IPCE spectra of the electrode prepared 
from the refluxed sol containing the dye (WE-r) and that steam-treated for $2 \mathrm{~h}$ (WE-rs). The thickness of the dye-containing layer of WE-r and WE-rs was not uniform, $700 \pm 200 \mathrm{~nm}$, and that slightly changed by the steam treatment. The layer consisted of 10-30 nm particles similar to those in the steam-treated electrodes as reported in our previous paper [14]. As the absorption spectrum of WE-r shown in Fig. 2(1) is compared to that of the untreated dye-doped titania gel electrode (WE) shown in Fig. 1(1), the peaks of these films are located around $460-480 \mathrm{~nm}$ depending on the acidity in the electrode. The main fluorescein species is the anion. The absorbance value of WE-r is ca. $15 \%$ lower than that of WE due to reflecting their dye-contents and the small difference between the molar extinction coefficients of the fluorescein species [28]. The number of the dye molecules loaded in WE-r was estimated to be ca. $20 \%$ lower than that in WE even though the dye-containing layer of WE-r was ca. 2 times as thick as that of WE. The amount of the dye encapsulated in or adsorbed on the titania nanoparticles in WE-r was expected to be smaller than that of WE because the refluxed sol heterogeneously contained dye and titania nanoparticles, in which the dye dispersion was lower than in the untreated sol. The effective IPCE values of the WE-r are ca. 2 times higher than those of WE when considering the dye content. The peaks of their photocurrent spectra are located at the longer wavelength, indicating a slight interaction 
and linkage between the carboxyl group of fluorescein and the titanium species. The photo-electric conversion performance was improved because the titania alkoxide molecules in the sol were polymerized and grew the nanocrystalline particles as the sol-gel reaction proceeded by reflux. The dye dispersion is expected to be high because the processes of the titania crystallization and the interaction between the titania and the dye molecules compete during the reflux compared to the conventional dye-adsorbed titania system. Instead of IPCE, the quantum efficiency of the working electrode samples will be discussed later to compare between the photoelectric properties of those different in the dye-content and the film thickness.

As WE-r was steam-treated, the absorbance decreased to ca. $10 \%$ of the initial value due to the dye desorption and the IPCE values tripled. Consequently, the IPCE values of WE-rs became similar to those of WE-s. The absorption band was somewhat red-shifted by an increase in the number of the dye-titania interaction species although the main fluorescein species in WE-r was different from in WE. The photo-electric conversion performance of the dye-doped titania electrode was improved using the refluxed titania sol. However, the effect was lower than the steam treatment.

(Figure 2) 


\subsection{Crystallinity of titania}

Figure 3 shows the XRD patterns of the untreated dye-doped titania gel film (WE), the steam-treated dye-doped titania film (WE-s), the films prepared from the refluxed sol (WE-r), and the films prepared from the refluxed sol and then steam-treated (WE-rs). No peak is found in the XRD pattern of the untreated amorphous gel film. An anatase type crystal was produced in the steam-treated film and the film prepared from the reflux sol. The size of the crystalline particles of these electrodes was estimated to be almost the same, i.e., $4.0-5.0 \mathrm{~nm}$, from their full-width at half-maximum of the $25^{\circ}$ peak using Sherrer's equation, $\mathrm{D}=0.9 \lambda / \beta \cdot \cos \theta$. The crystal size of WE-r was only slightly changed by the steam treatment based on the XRD analysis.

\section{(Figure 3)}

Figure 4 and Table 1 show the $I-V$ curve and the photo-electric conversion properties along with the quantum efficiency for each electrode sample, respectively. The short circuit photocurrent density $\left(J_{\mathrm{SC}}\right)$ and open circuit voltage $\left(V_{\mathrm{OC}}\right)$ values of the steam-treated electrodes (WE-s) are significantly higher than the others due to the higher values of its photocurrent spectrum as shown in Figure 1. The steam treatment also improved the fill factor $(F F)$, the maximum power $\left(P_{\max }\right)$, and quantum efficiency $(Q E)$, i.e. absorbed photons to current conversion efficiency, of the cell. The $J_{\mathrm{SC}}$ 
values of the electrodes prepared from the reflux sols (WE-r) are higher than those of the gel film (WE). The $V_{\mathrm{OC}}$ values of WE-r, however, are lower than those of WE, leading to the lower $F F$ value of WE-r. This is expected due to the remarkable charge recombination between the dye and the nanocrystalline titania. All the parameters of the photo-electric conversion were improved by the steam treatment based on the values for WE-s and WE-rs. The IPCE values of WE, WE-s, WE-r, and WE-rs at $350 \mathrm{~nm}$ were $1.6 \%, 5.2 \%, 5.3$ and $4.8 \%$, respectively, mainly depending on their crystallinity. The last three are regarded as having a close crystallinity. The IPCE values in the visible range of WE-r were lower than those of WE-s as shown in Figs. 1 and 2 even though the UV photocurrent generated from the titanium nanocrystalline part of WE-r should be almost same as that of WE-s. The photovoltaic performance of the dye-doped titania of WE-s during visible irradiation was better than that of WE-r, indicating that the efficiency of the electron injection of WE-s was remarkably higher than that of WE-r. The steam treatment enhanced all the parameters for WE-r, especially the $V_{\mathrm{OC}}$ value, i.e., the value of WE-rs was close to that of WE-s. This is because the charge recombination was restrained by the enhancement of the dye-titania interaction.

The quantum efficiency of the dye-doped titania electrode, that is, the performance 
of the cell, was improved by the steam treatment and using the refluxed titania sol.

The quantum efficiency is useful value to examine the effective dye sensitization of the working electrodes which are different in the dye content and the film thickness. The efficiency for WE and WE-r increased by roughly 30 and 10 times higher after the 2-h steam treatment, respectively. The electrode prepared from the refluxed sol (WE-r) also exhibited about a 10 times higher performance than the untreated amorphous sample (WE). The difference in the quantum efficiency strongly depends on the electron transfer from the dye to the titania, influenced by the dye dispersion in the titania, rather than the characteristics of the nanocrystalline titania such as its crystalinity and particle size. The steam treatment after the preparation of the dye-doped gel films is sure to promote the dye-titania interaction.

(Figure 4 and Table 1)

\subsection{Dye-titania interaction}

The spectral shape of each electrode sample was compared in Figure 5. The photocurrent spectrum was red-shifted by the steam-treatment, indicating that the interaction of the dye and titania were enhanced and the electron injection efficiency improved. The present experimental results indicate that the crystallinity of the dye-doped titania layer influences the conversion efficiency due to an enhancement of 
the electric conductivity. Not only this, but also the electron injection process from the dye to the titania are important for the photo-electric conversion efficiency. The dye-titania interaction in the WE-s is better than that in the WE-r due to the dye dispersion into the titania matrix without any dye aggregation during the room temperature sol-gel process. The steam treatment was carried out after the dye-dispersion, whereas the crystallization by refluxing the dye-containing sol somewhat decreased the dye dispersion. Therefore, the steam treatment enhances not only the density of the titania particles, but also the dye-titania interaction, and they play an important role in transporting the electron through the electrode $[14,15]$. The steam treatment of WE-r allowed the dye molecules to significantly interact with the titanium species. Active gaseous water promoted the hydrolysis of the titanium species and enhanced their reactivity. We strongly suggest that the fluorescein molecules formed a chelate complex with the titanium species on the titania surface to induce the interaction between their orbitals in the steam-treated electrodes based on the spectral red-shift. This interaction caused the ligand to metal charge transfer (LMCT) interaction and the fast electron injection to the titania conduction band $[8,9,16,30]$. It is suggested that the fluorescein dianion is the preferential species for titanium chelation due to the nucleophilicity of its carboxylate. The $V_{\mathrm{OC}}$ values were increased 
by the steam treatment because the chelate complex formation caused the negative shift of the conduction band potential. [30]

(Figure 5)

\section{Conclusions}

The influences of the titania nanostructure and dye dispersion in the dye-doped titania electrodes on its photo-electric conversion property were revealed by simple spectroscopic and electric measurements. In order to obtain differences in the titania nanostructure and the dye dispersion, (1) the dye-doped titania gel films were prepared from the titanium alkoxide solution containing the dye and then steam-treated, and (2) the-doped titania films were prepared from the refluxed titanium alkoxide sol containing the dye. The photocurrent quantum efficiency of the electrodes remarkably increased by (1) the steam treatment of the electrodes and (2) using the refluxed titania sol to prepare the electrodes, compared to that of the untreated dye-doped electrode consisting of amorphous titania gel. The growth and crystallization of the titania particles and the decrease in defect density by the steam treatment and reflux improved the electric conductivity. The efficiency in the former was higher than that in the latter due to the dye dispersion into the titania matrix. The steam treatment was carried out after the 
dye molecules were highly dispersed without any dye aggregation during the room temperature sol-gel process, whereas the crystallization by refluxing the dye-containing sol somewhat decreased the dye dispersion. It is suggested that the steam treatment is an effective method to enhance not only the electric conductivity of the titania depending on its nanostructure, but also the dye-titania interaction depending on the dye dispersion, playing an important role in the transport of the electron through the electrode. The steam treatment promoted the formation of the dye-titanium LMCT complex on the titania surface, inducing the fast electron injection to the titania conduction band.

Acknowledgements

This work was supported by the Sasagawa Scientific Research Grant from The Japan Science Society, 1997-1998, the Research Grant from The Thermal \& Electric Energy Technology Foundation, 2001, and the Ministry of Education, Science, Sports and Culture, Grant-in-Aid for Young Scientists (B), 2008.

References 
[1] B. O’Regan, M. Grätzel, Nature 353 (1991) 737.

[2] M. K. Nazeeruddin, A. Kay, I. Rodicio, R. Hamphry-Baker, E. Müeller, P. Liska, N. Vlachopoulos, M. Grätzel, J. Am. Chem. Soc. 115 (1993) 6382.

[3] M. Grätzel, J. Photochem. Photobiol. C: Photochem. Rev. 4 (2003) 145.

[4] K. Murakoshi, G. Kano, Y. Wada, S. Yanagida, H. Miyazaki, M. Matsumoto, S. Murasawa, J. Electroanal. Chem. 396 (1995) 27.

[5] K. Kalyanasundaram, M. Grätzel, Coordination Chem. Rev. 177 (1998) 347.

[6] J. He, F. Chen, J. Zhao, H. Hidaka, Colloids Surfaces A: Physicochem. Eng. Aspects 142 (1998) 49.

[7] C. Wang, C. Liu, Y. Wang, T. Shen, J. Colloid Interface Sci. 197 (1998) 126.

[8] M. Hilgendorff, V. Sundström, J. Phys. Chem. B 102 (1998) 10505.

[9] G. Ramakrishna, H. N. Ghosh, J. Phys. Chem. B 105 (2001) 7000.

[10] G. Benkö, M. Hilgendorff, A. P. Yartsev, V. Sundström, J. Phys. Chem. B 105 (2001) 967.

[11] G. Benkö, B. Skårman, R. Wallenberg, A. Hagfeldt, V. Sundström, A. P. Yartsev, J. Phys. Chem. B 107 (2003) 1370.

[12] S. Pelet, M. Grätzel, J. E. Moser, J. Phys. Chem. B 107 (2003) 3215.

[13] G. Ramakrishna, A. Das, H. N. Ghosh, Langmuir 20 (2004) 1430. 
[14] H. Nishikiori, N. Tanaka, T. Kitsui, T. Fujii, J. Photochem. Photobiol. A: Chem. 179 (2006) 125.

[15] T. Kitsui, H. Nishikiori, N. Tanaka, T. Fujii, J. Photochem. Photobiol. A: Chem. $192(2006) 220$.

[16] H. Nishikiori, W. Qian, M.A. El-Sayed, N. Tanaka, T. Fujii, J. Phys. Chem. C 111 (2007) 9008.

[17] H. Itai, H. Nishikiori, N. Tanaka, M. Endo, T Fujii, Chem. Lett. 37 (2008) 940.

[18] S. Yamazaki-Nishida, K. J. Nagano, L. A. Philips, S. Cervera-March, M. A. Anderson, J. Photochem. Photobiol. A: Chem. 70 (1993) 95.

[19] Y. Djaoued, S. Badilescu, P. V. Ashrit, D. Bersani, P. P. Lottici, R. Brüning, J. Sol-Gel Sci. Technol. 24 (2002) 247.

[20] Y. J. Yun, J. S. Chung, S. Kim, S. H. Hahn, E. J. Kim, Mater. Lett. 58 (2004) 3703.

[21] Y. Hu, C. Yuan, J. Crystal Growth 274 (2005) 563.

[22] L. Wu, J. C. Yu, X. Wang, L. Zhang, J. Yu, J. Solid State Chem. 178 (2005) 321.

[23] G. Li, L. Li, J. Boerio-Goates, .B. F. Woodfield, J. Am. Chem. Soc. 127(2005) 8659.

[24] L. Ge, M. Xu, M. Sun, H. Fang, J. Sol-Gel Sci. Technol. 38 (2006) 47. 
[25] M. J. Jensen, P. A. Fuierer, J. Sol-Gel Sci. Technol. 39 (2006) 229.

[26] T. Sato, Y. Aita, M. Komatsu, S. Yin, J. Mater. Sci. 41 (2006) 1433.

[27] R. K. Wahi, Y. Liu, J. C. Falkner, V. L. Colvin, J. Colloid Interface Sci. 302 (2006) 530.

[28] T. Fujii, A. Ishii, N. Tagusagawa, Res. Chem. Intermed. 17 (1992) 1.

[29] J. N. O’Shea, J. B. Taylor, E. F. Smith, Surf. Sci. 548 (2004) 317.

[30] D. EL Mekkawi, M. S. A. Abdel-Mottaleb, Int. J. Photoenergy 7 (2005) 95. 
Figure captions

Figure 1. Visible absorption and IPCE spectra of the dye-doped titania gel electrode before, WE (1), and after, WE-s (2), the steam treatment.

Figure 2. Visible absorption and IPCE spectra of the electrode prepared from the refluxed sol, WE-r (1), and that steam-treated, WE-rs (2).

Figure 3. XRD patterns of the untreated titania gel film, WE (1), the steam-treated titania gel film, WE-s (2), the film prepared from the refluxed sol, WE-r (3), and the film that was prepared from the refluxed sol and then steam-treated, WE-rs (4).

Figure 4. Photo-electric conversion properties of the untreated titania gel electrode, WE (1), the steam-treated titania gel electrode, WE-s (2), the electrode prepared from the refluxed sol, WE-r (3), and the electrode that was prepared from the refluxed sol and then steam-treated, WE-rs (4).

Figure 5. Shape of photocurrent spectra of the untreated titania gel electrode, WE, 
the steam-treated titania gel electrode, WE-s, the electrode prepared from the refluxed sol, WE-r, and the electrode that was prepared from the refluxed sol and then steam-treated, WE-rs. 
Figure 1

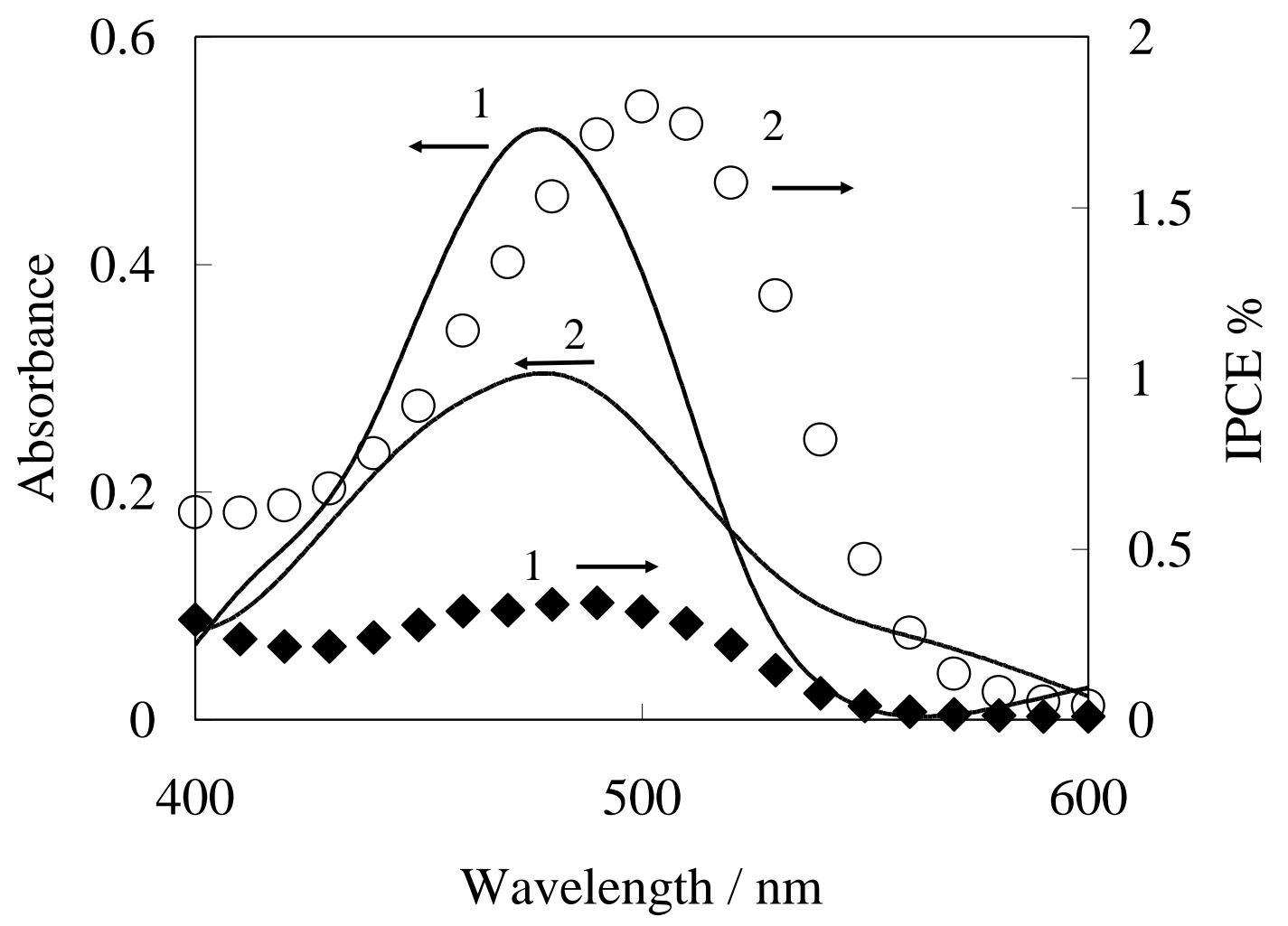


Figure 2

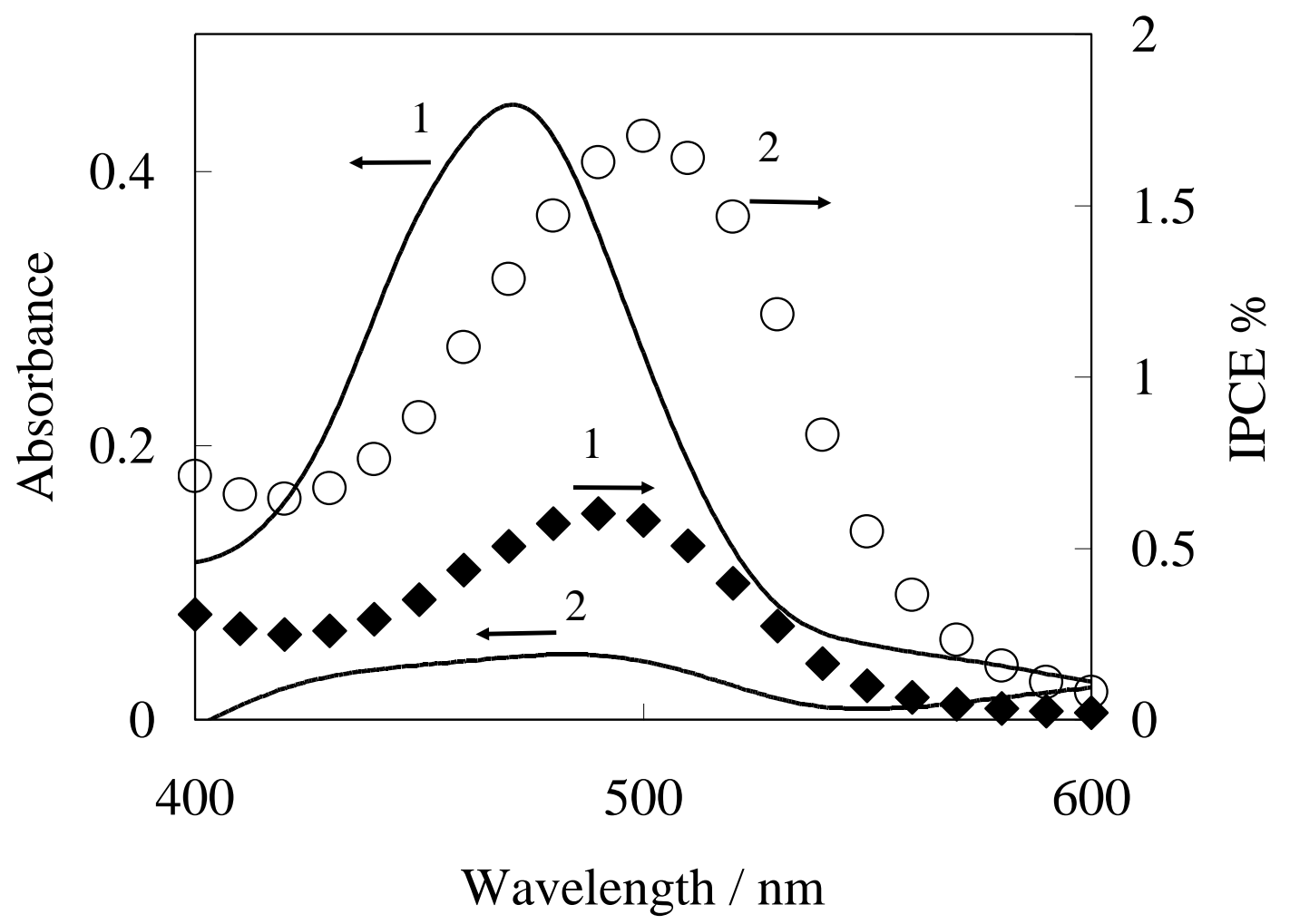


Figure 3

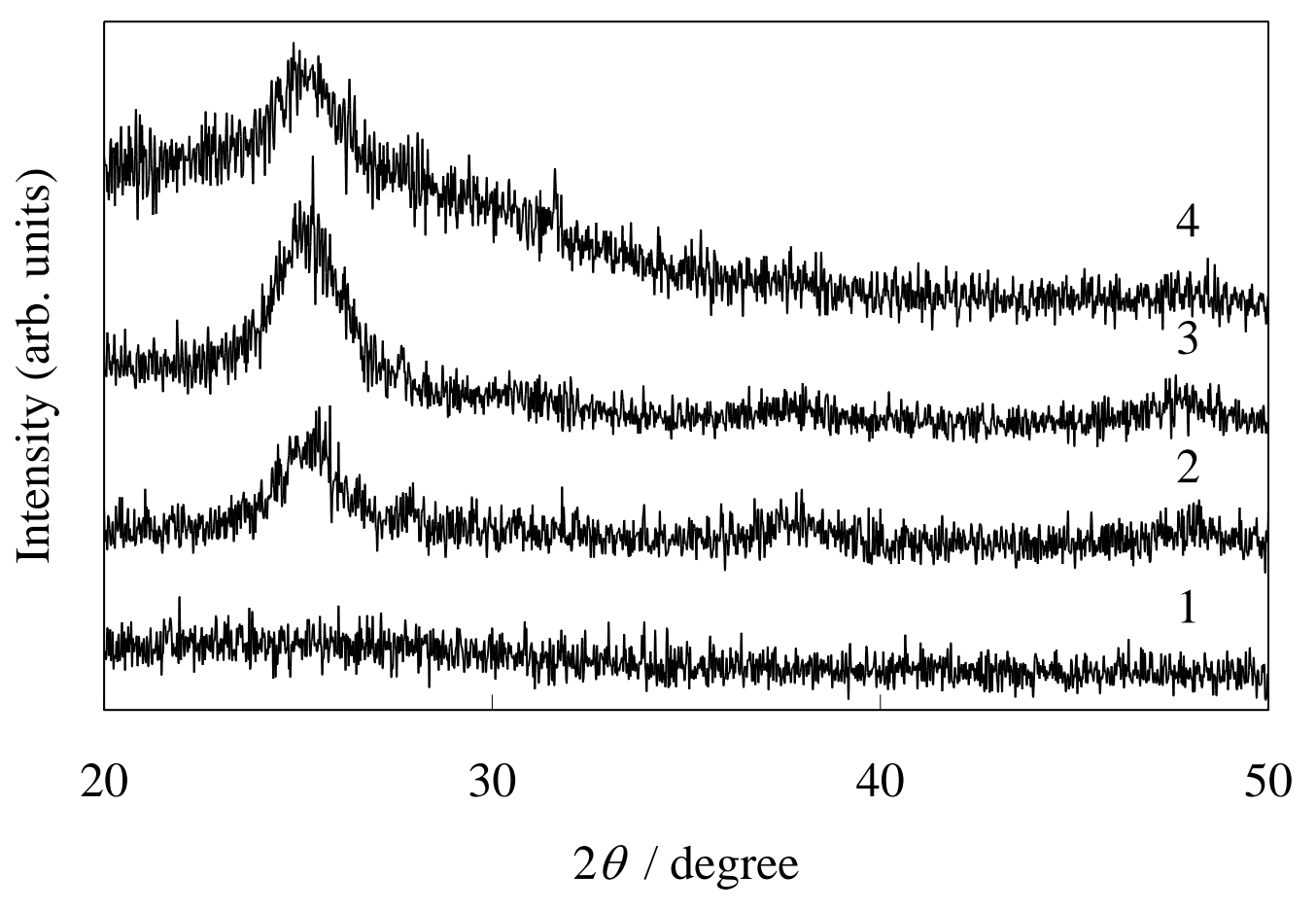


Figure 4

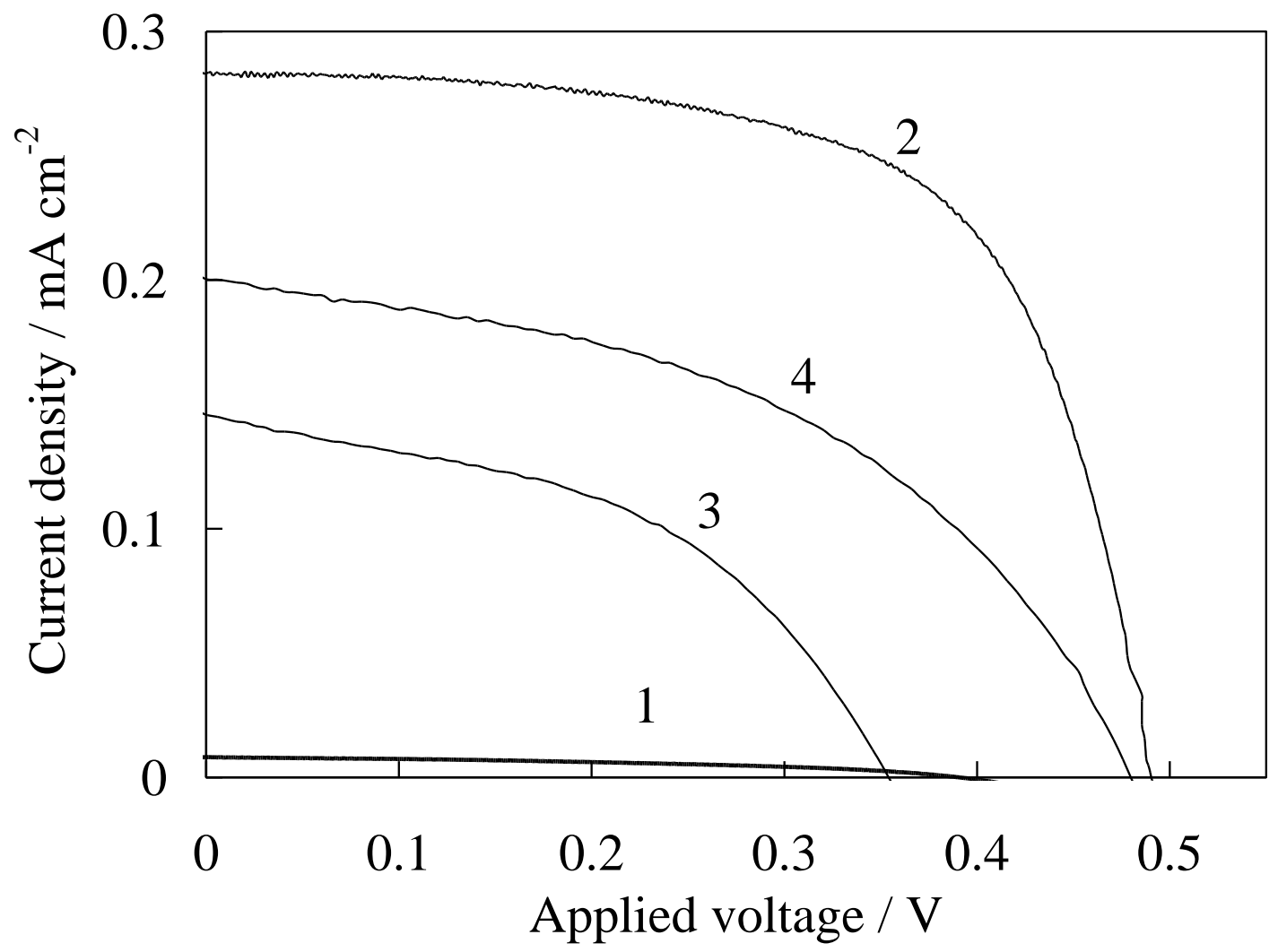


Figure 5

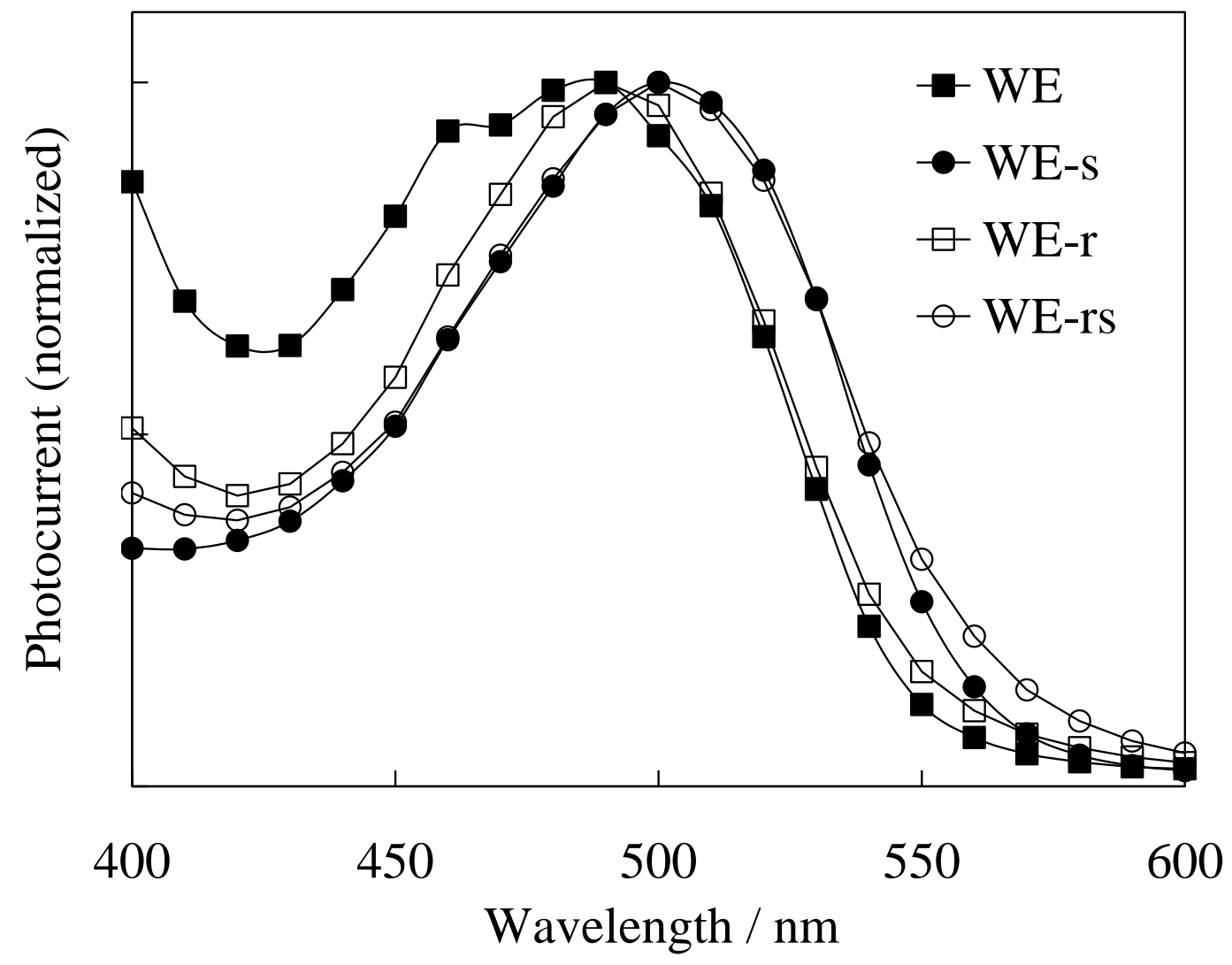


Table 1 Photo-electric conversion properties of each electrode sample.

\begin{tabular}{cccccc}
\hline Sample & $\begin{array}{c}J_{\mathrm{SC}} \\
/ \mathrm{mA} \mathrm{cm}^{-2}\end{array}$ & $V_{\mathrm{OC}} / \mathrm{V}$ & $F F$ & $\begin{array}{c}P_{\max } / \\
\mu \mathrm{W} \mathrm{cm}{ }^{-2}\end{array}$ & $Q E / \%$ \\
\hline WE & 0.0082 & 0.39 & 0.44 & 1.4 & 0.13 \\
WE-s & 0.28 & 0.49 & 0.64 & 89 & 4.0 \\
WE-r & 0.15 & 0.36 & 0.45 & 24 & 1.5 \\
WE-rs & 0.20 & 0.48 & 0.47 & 45 & 19 \\
\hline
\end{tabular}

$J_{\mathrm{SC}}$ : short circuit photocurrent density

$V_{\mathrm{OC}}:$ open circuit voltage

$F F:$ fill factor

$P_{\max }:$ maximum power

$Q E:$ quantum efficiency at $500 \mathrm{~nm}$ 